\title{
Raman Line Imaging of \\ Poly(E-caprolactone)/Carbon Dioxide Solutions at High Pressures: \\ A Combined Experimental and Computational Study for Interpreting Intermolecular Interactions and Free-Volume Effects
}

\author{
Maria Giovanna Pastore Carbone ${ }^{1}$, Pellegrino Musto ${ }^{2 *}$, Marianna Pannico ${ }^{2}$, Andreas Braeuer ${ }^{3}$, Giuseppe \\ Scherillo ${ }^{1}$, Giuseppe Mensitieri ${ }^{1}$ and Ernesto Di Maio ${ }^{1}$ \\ ${ }^{1}$ Department of Chemical, Materials and Production Engineering, University of Naples Federico \\ II Institute on Polymers Composites and Biomaterials, National Research Council of Italy, Italy; \\ ${ }^{3}$ Erlangen Graduate School in Advanced Optical Technologies (SAOT) and Lehrstuhl für Technische \\ Thermodynamik (LTT), Friedrich-Alexander-Universität Erlangen-Nürnberg, Germany.
}

\section{Supplementary Data}

Contents:

Page 2: $\quad$ Figure S1 - Initial configurations of the donor-acceptor complexes.

Figure S2 - Atom numbering for the isolated pentylhexanoate (PHEX) molecule.

Table S1 - Definition of internal coordinates and PED for the isolated PHEX molecule.

Page 5: $\quad$ Figure $\mathrm{S} 3$ - Atom numbering for the $\mathrm{PHEX} / \mathrm{CO}_{2}$ complex from the alkyl-side starting structure.

Table S2 - Definition of internals and PED for the PHEX/CO $\mathrm{CO}_{2}$ complex from the alkylside starting structure.

Page 8: $\quad$ Figure $\mathrm{S} 4$ - Atom numbering for the PHEX/CO $/ \mathrm{C}_{2}$ complex from the ester-side starting structure.

Table S3 - Definition of internals and PED for the PHEX/CO 2 complex from the ester-side starting structure.

Page 10: Figure $\mathrm{S} 5$ - Atom numbering for the $\mathrm{PHEX} / \mathrm{CO}_{2}$ complex from the $\mathrm{sp}^{3}-\mathrm{O} / \mathrm{CO}_{2}$ starting structure.

Table S3 - Definition of internals and PED for the PHEX/CO $/ \mathrm{O}_{2}$ complex from the $\mathrm{sp}^{3}$ $\mathrm{O} / \mathrm{CO}_{2}$ starting structure. 


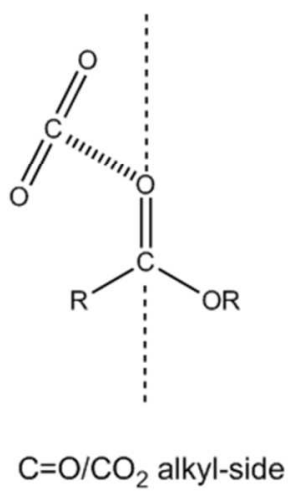

A
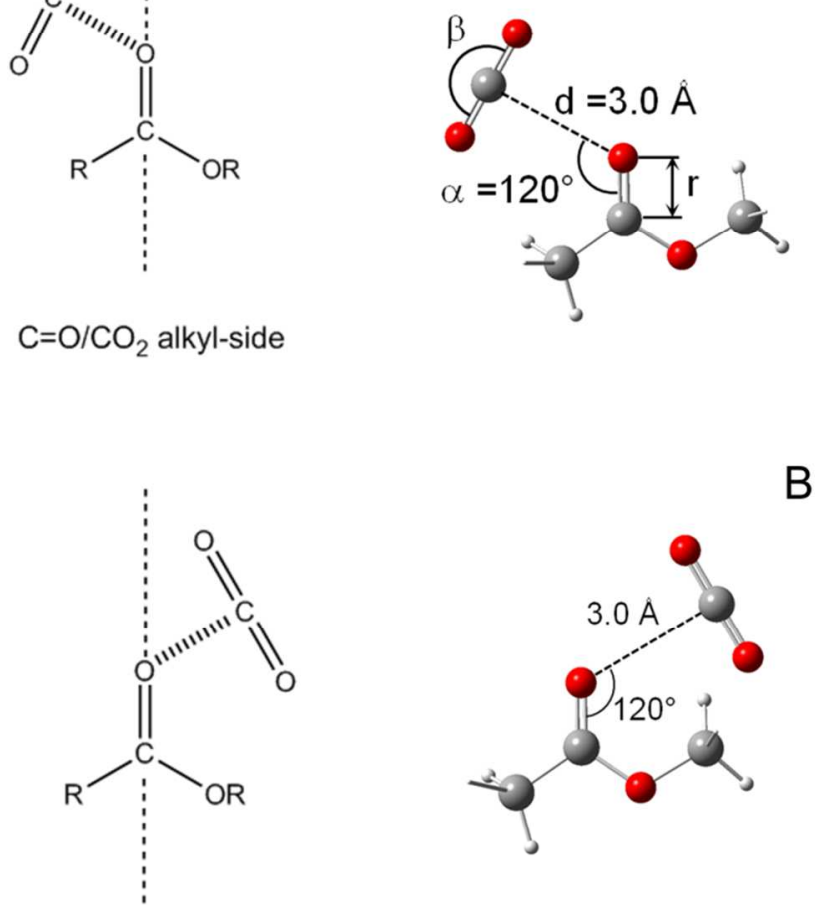

$\mathrm{C}=\mathrm{O} / \mathrm{CO}_{2}$ ester-side

B

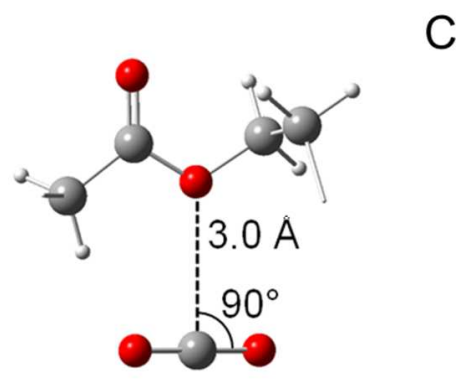

Figure s1. Starting-point structures for the optimization of the supramolecular aggregate. A) $>\mathrm{C}=\mathrm{O} / \mathrm{CO}_{2}$ alkyl-side; $>\mathrm{C}=\mathrm{O} / \mathrm{CO}_{2}$ ester-side; $\left.\mathrm{C}\right) \mathrm{sp}^{3} \mathrm{O} / \mathrm{CO}_{2}$. 


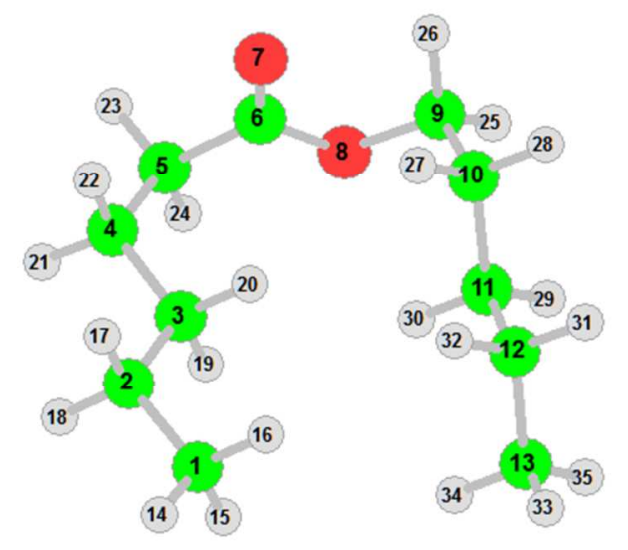

Figure S2. Atom numbering for the isolated pentylhexanoate (PHEX) molecule. Atom colour code: Green $=C$; grey=H; red=O.

Table s1. Definition of internal coordinates (natural) and their main contributions to the PED (values $\leq 10$ o neglected) for the isolated pentylhexanoate (PHEX) molecule. The indicated frequencies are unscaled.

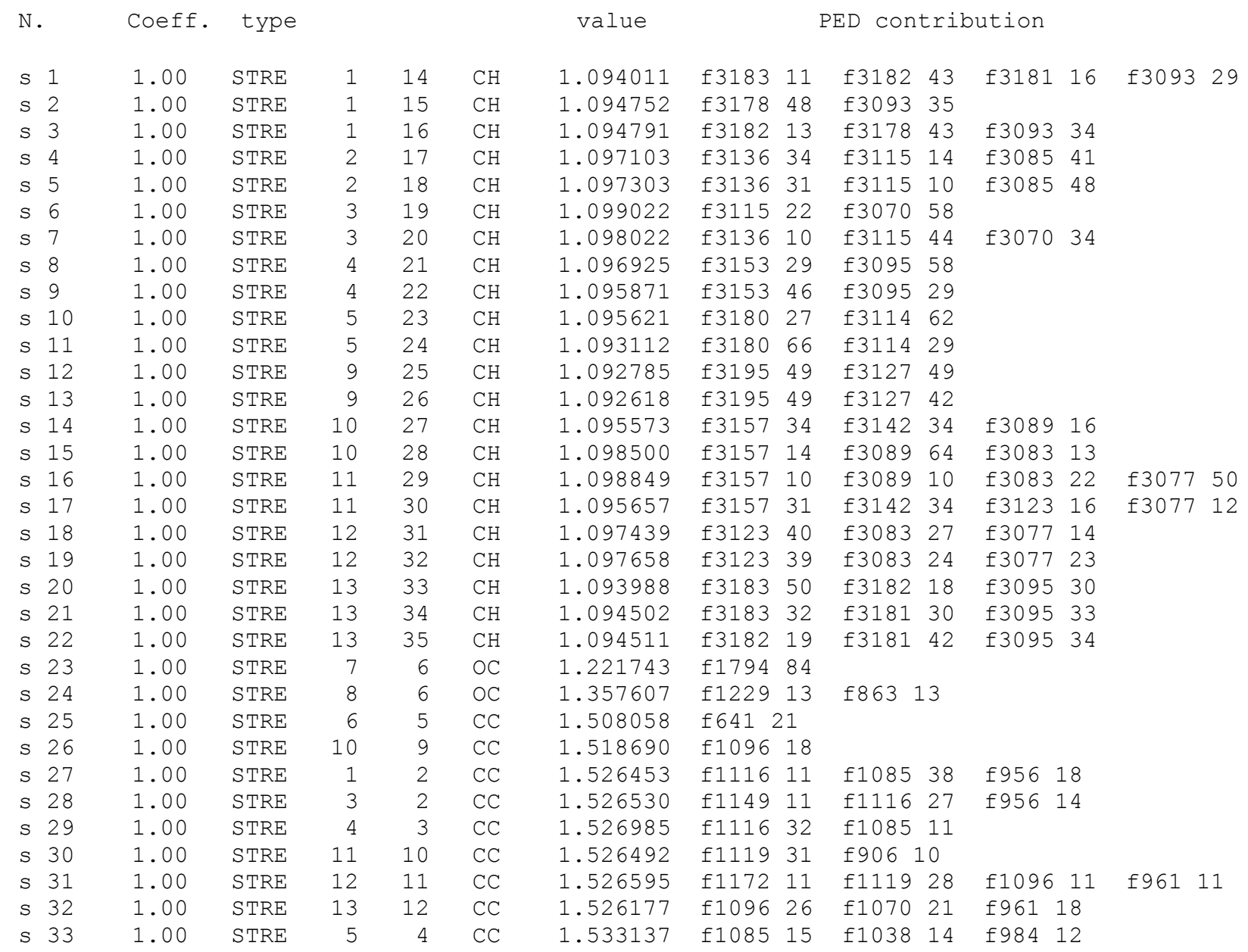




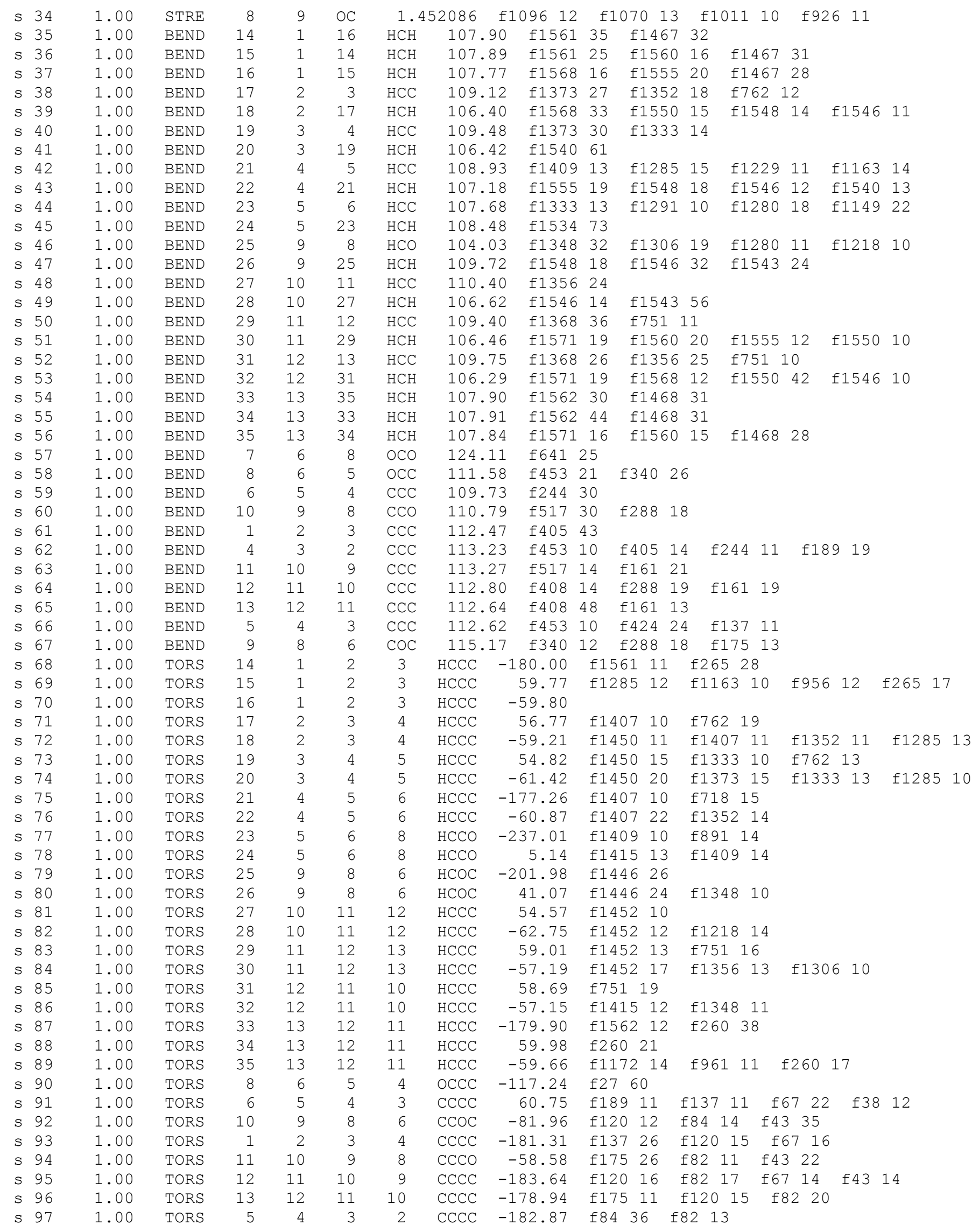




$\begin{array}{llllllllrllllll}\mathrm{S} & 98 & 1.00 & \text { TORS } & 9 & 8 & 6 & 5 & \text { COCC } & -183.81 & f 120 & 13 & f 38 & 42 & \\ \text { S } 99 & 1.00 & \text { OUT } & 7 & 5 & 8 & 6 & \text { OCOC } & 1.52 & f 863 & 10 & f 718 & 28 & f 641 & 14\end{array}$

34 STRETCHING modes:

$\begin{array}{llllllllllllllllllll}1 & 2 & 3 & 4 & 5 & 6 & 7 & 8 & 9 & 10 & 11 & 12 & 13 & 14 & 15 & 16 & 17 & 18 & 19 & 20\end{array}$

$\begin{array}{llllllllllllll}21 & 22 & 23 & 24 & 25 & 26 & 27 & 28 & 29 & 30 & 31 & 32 & 33 & 34\end{array}$

33 BENDING modes:

$\begin{array}{llllllllllllllllllll}35 & 36 & 37 & 38 & 39 & 40 & 41 & 42 & 43 & 44 & 45 & 46 & 47 & 48 & 49 & 50 & 51 & 52 & 53 & 54\end{array}$

$\begin{array}{lllllllllllll}55 & 56 & 57 & 58 & 59 & 60 & 61 & 62 & 63 & 64 & 65 & 66 & 67\end{array}$

32 TORSION modes:

$\begin{array}{llllllllllllllllllll}68 & 69 & 70 & 71 & 72 & 73 & 74 & 75 & 76 & 77 & 78 & 79 & 80 & 81 & 82 & 83 & 84 & 85 & 86 & 87\end{array}$

$\begin{array}{llllllllllll}88 & 89 & 90 & 91 & 92 & 93 & 94 & 95 & 96 & 97 & 98 & 99\end{array}$

1 OUT-OF-PLANE BENDING (WAGGING) MODE

$66 \mathrm{CH}$ modes:

$\begin{array}{llllllllllllllllllll}1 & 2 & 3 & 4 & 5 & 6 & 7 & 8 & 9 & 10 & 11 & 12 & 13 & 14 & 15 & 16 & 17 & 18 & 19 & 20\end{array}$

$\begin{array}{llllllllllllllllllll}21 & 22 & 35 & 36 & 37 & 38 & 39 & 40 & 41 & 42 & 43 & 44 & 45 & 46 & 47 & 48 & 49 & 50 & 51 & 52\end{array}$

$\begin{array}{llllllllllllllllllll}53 & 54 & 55 & 56 & 68 & 69 & 70 & 71 & 72 & 73 & 74 & 75 & 76 & 77 & 78 & 79 & 80 & 81 & 82 & 83\end{array}$

$\begin{array}{llllll}84 & 85 & 86 & 87 & 88 & 89\end{array}$

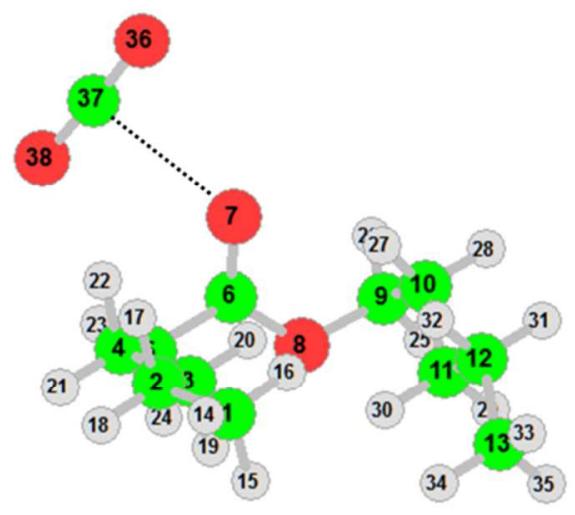

Figure s3. Atom numbering for the pentylhexanoate/ $\mathrm{CO}_{2}$ complex from the alkyl-side starting structure. Atom colour code: Green=C; grey=H; red=O.

Table s2. Definition of internal coordinates (natural) and their main contributions to the PED (values $\leq 10$ \% neglected) for the pentylhexanoate/ $\mathrm{H}_{2} \mathrm{O}$ complex from the alkyl-side starting structure. The indicated frequencies are unscaled.

\begin{tabular}{|c|c|c|c|c|c|c|c|c|c|c|c|c|c|}
\hline N. & Coeff. & type & & & & value & & & ED con & trik & ution & & \\
\hline s 1 & 1.00 & STRE & 1 & 14 & $\mathrm{CH}$ & 1.094006 & $\mathrm{f} 3180$ & 18 & f3179 & 36 & f3178 & 14 & f3091 29 \\
\hline s 2 & 1.00 & STRE & 1 & 15 & $\mathrm{CH}$ & 1.094758 & f3176 & 52 & £3091 & 35 & & & \\
\hline s 3 & 1.00 & STRE & 1 & 16 & $\mathrm{CH}$ & 1.094791 & f3179 & 14 & f3176 & 39 & f3091 & 33 & \\
\hline s 4 & 1.00 & STRE & 2 & 17 & $\mathrm{CH}$ & 1.097155 & f3133 & 33 & f3113 & 16 & f3083 & 43 & \\
\hline 5 & 1.00 & STRE & 2 & 18 & $\mathrm{CH}$ & 1.097263 & f3133 & 32 & f3113 & 11 & f3083 & 48 & \\
\hline
\end{tabular}




\begin{tabular}{|c|c|c|c|c|c|c|c|c|c|c|c|c|c|c|c|c|c|}
\hline 3 & 6 & 1.00 & STRE & 3 & 19 & $\mathrm{CH}$ & \multicolumn{2}{|c|}{1.098998} & f3113 22 & \multicolumn{3}{|c|}{ f3068 57} & & & & \\
\hline $\mathrm{s}$ & 7 & 1.00 & STRE & 3 & 20 & $\mathrm{CH}$ & 1.0 & 8123 & f3133 13 & & 1134 & 45 & f3068 & $3 \quad 35$ & & & \\
\hline S & 8 & 1.00 & STRE & 4 & 21 & $\mathrm{CH}$ & 1.0 & 6885 & f3156 23 & & 0966 & 68 & & & & & \\
\hline S & 9 & 1.00 & STRE & 4 & 22 & $\mathrm{CH}$ & 1.0 & 5295 & f3156 50 & & 0962 & 24 & & & & & \\
\hline S & 10 & 1.00 & STRE & 5 & 23 & $\mathrm{CH}$ & 1.0 & 4694 & f3182 36 & & 1185 & 57 & & & & & \\
\hline s & 11 & 1.00 & STRE & 5 & 24 & $\mathrm{CH}$ & 1.0 & 3083 & f3182 58 & & 1184 & 40 & & & & & \\
\hline S & 12 & 1.00 & STRE & 9 & 25 & $\mathrm{CH}$ & 1.0 & 2680 & f319348 & & 1254 & 49 & & & & & \\
\hline$S$ & 13 & 1.00 & STRE & 9 & 26 & $\mathrm{CH}$ & 1.0 & 2446 & f3193 50 & & 1254 & 41 & & & & & \\
\hline S & 14 & 1.00 & STRE & 10 & 27 & $\mathrm{CH}$ & 1.0 & 5642 & f3154 30 & & 1403 & 36 & f3086 & 615 & & & \\
\hline S & 15 & 1.00 & STRE & 10 & 28 & $\mathrm{CH}$ & 1.0 & 8463 & f3154 12 & & 0866 & 63 & f3081 & 14 & & & \\
\hline S & 16 & 1.00 & STRE & 11 & 29 & $\mathrm{CH}$ & 1.0 & 8828 & f3154 10 & & 0861 & 10 & f3081 & 20 & f30 & $75 \quad 53$ & \\
\hline S & 17 & 1.00 & STRE & 11 & 30 & $\mathrm{CH}$ & 1.0 & 5656 & f3154 30 & & 1403 & 33 & f3121 & 16 & 130 & $75 \quad 12$ & \\
\hline S & 18 & 1.00 & STRE & 12 & 31 & $\mathrm{CH}$ & 1.0 & 7428 & f3121 39 & & 0812 & 29 & f3075 & $5 \quad 13$ & & & \\
\hline S & 19 & 1.00 & STRE & 12 & 32 & $\mathrm{CH}$ & 1.0 & 7634 & f3121 41 & & 0812 & 24 & f3075 & 521 & & & \\
\hline$S$ & 20 & 1.00 & STRE & 13 & 33 & $\mathrm{CH}$ & 1.0 & 3964 & f3180 37 & & 1792 & 29 & f3092 & 230 & & & \\
\hline S & 21 & 1.00 & STRE & 13 & 34 & $\mathrm{CH}$ & 1.0 & 4463 & f3180 35 & & 1782 & 27 & f3092 & 233 & & & \\
\hline s & 22 & 1.00 & STRE & 13 & 35 & $\mathrm{CH}$ & 1.0 & 4503 & f3179 16 & & 1784 & 46 & f3092 & 235 & & & \\
\hline S & 23 & 1.00 & STRE & 36 & 37 & OC & 1.1 & 7861 & f2403 51 & & 3214 & 49 & & & & & \\
\hline s & 24 & 1.00 & STRE & 38 & 37 & OC & 1.1 & 1206 & f2403 49 & & 3215 & 51 & & & & & \\
\hline S & 25 & 1.00 & STRE & 7 & 6 & $\mathrm{OC}$ & 1.2 & 4609 & f1776 83 & & & & & & & & \\
\hline S & 26 & 1.00 & STRE & 8 & 6 & OC & 1.3 & 4077 & f1307 10 & & 2301 & 12 & f863 & 14 & & & \\
\hline S & 27 & 1.00 & STRE & 6 & 5 & $\mathrm{CC}$ & 1.5 & 6205 & f643 22 & & & & & & & & \\
\hline S & 28 & 1.00 & STRE & 10 & 9 & $\mathrm{CC}$ & 1.5 & 8269 & f1095 18 & & & & & & & & \\
\hline S & 29 & 1.00 & STRE & 1 & 2 & $\mathrm{CC}$ & 1.5 & 6417 & $f 111510$ & & 0853 & 38 & f956 & 18 & & & \\
\hline S & 30 & 1.00 & STRE & 3 & 2 & $\mathrm{CC}$ & 1.5 & 6572 & $f 1150 \quad 10$ & & 1152 & 28 & f956 & 14 & & & \\
\hline S & 31 & 1.00 & STRE & 4 & 3 & $\mathrm{CC}$ & 1.5 & 6783 & $f 111533$ & & 0851 & 10 & & & & & \\
\hline S & 32 & 1.00 & STRE & 11 & 10 & $\mathrm{CC}$ & 1.5 & 6511 & $f 1171 \quad 10$ & & 1183 & 31 & & & & & \\
\hline S & 33 & 1.00 & STRE & 12 & 11 & $\mathrm{CC}$ & 1.5 & 6589 & f1171 11 & & 1182 & 28 & f1095 & $5 \quad 11$ & f96 & 011 & \\
\hline S & 34 & 1.00 & STRE & 13 & 12 & $\mathrm{CC}$ & 1.5 & 6158 & f1095 26 & & 0692 & 20 & f960 & 18 & & & \\
\hline s & 35 & 1.00 & STRE & 5 & 4 & $\mathrm{CC}$ & 1.5 & 3795 & f1085 15 & & 0381 & 14 & f983 & 11 & & & \\
\hline S & 36 & 1.00 & STRE & 8 & 9 & OC & 1.4 & 3513 & f1095 11 & & 0691 & 13 & f925 & 10 & f906 & 10 & \\
\hline s & 37 & 1.00 & STRE & 7 & 37 & OC & 2.7 & 8970 & f107 10 & f89 & 13 & f80 & 47 & & & & \\
\hline s & 38 & 1.00 & BEND & 14 & 1 & 16 & $\mathrm{HCH}$ & 107.90 & $0 \quad f 1560$ & 35 & f146 & 1663 & 32 & & & & \\
\hline S & 39 & 1.00 & BEND & 15 & 1 & 14 & $\mathrm{HCH}$ & 107.88 & f1560 & 24 & f156 & 5601 & $\mathrm{f} 1$ & 466 & 31 & & \\
\hline S & 40 & 1.00 & BEND & 16 & 1 & 15 & $\mathrm{HCH}$ & 107.77 & f1567 & 15 & f155 & 551 & $\mathrm{f} 1$ & 466 & 28 & & \\
\hline S & 41 & 1.00 & BEND & 17 & 2 & 1 & $\mathrm{HCC}$ & 109.67 & f1372 & 23 & f135 & 3502 & f7 & 7631 & 11 & & \\
\hline s & 42 & 1.00 & BEND & 18 & 2 & 17 & $\mathrm{HCH}$ & 106.39 & f1567 & 32 & f 154 & 491 & $\mathrm{fl}$ & 547 & 14 & & \\
\hline s & 43 & 1.00 & BEND & 19 & 3 & 4 & $\mathrm{HCC}$ & 109.52 & f1372 & 29 & f133 & 341 & 3 & & & & \\
\hline S & 44 & 1.00 & BEND & 20 & 3 & 19 & $\mathrm{HCH}$ & 106.39 & f1539 & 62 & & & & & & & \\
\hline S & 45 & 1.00 & BEND & 21 & 4 & 5 & $\mathrm{HCC}$ & 108.65 & f1408 & 13 & $\mathrm{f} 128$ & 841 & $\mathrm{f} 1$ & 1230 & 10 & f11 62 & 15 \\
\hline S & 46 & 1.00 & BEND & 22 & 4 & 21 & $\mathrm{HCH}$ & 106.92 & f1555 & 22 & f 154 & 5491 & $\mathrm{f} 1$ & 547 & 17 & f1539 & 12 \\
\hline S & 47 & 1.00 & BEND & 23 & 5 & 6 & $\mathrm{HCC}$ & 107.73 & f1334 & 17 & $\mathrm{f} 129$ & 931 & $\mathrm{f} 1$ & 150 & 21 & & \\
\hline s & 48 & 1.00 & BEND & 24 & 5 & 23 & $\mathrm{HCH}$ & 108.87 & f1532 & 73 & & & & & & & \\
\hline S & 49 & 1.00 & BEND & 25 & 9 & 8 & $\mathrm{HCO}$ & 103.96 & f1347 & 30 & $\mathrm{f} 130$ & 3071 & $\mathrm{f} 1$ & 1282 & 10 & f1217 & 10 \\
\hline S & 50 & 1.00 & BEND & 26 & 9 & 25 & $\mathrm{HCH}$ & 109.76 & f1547 & 12 & f 154 & $54 \quad 4$ & $\mathrm{f} 1$ & 541 & 20 & & \\
\hline s & 51 & 1.00 & BEND & 27 & 10 & 11 & $\mathrm{HCC}$ & 110.47 & f1356 & 24 & $\mathrm{f} 128$ & 821 & 1 & & & & \\
\hline S & 52 & 1.00 & BEND & 28 & 10 & 27 & $\mathrm{HCH}$ & 106.58 & f1545 & 13 & f154 & 545 & 59 & & & & \\
\hline S & 53 & 1.00 & BEND & 29 & 11 & 12 & $\mathrm{HCC}$ & 109.38 & f1368 & 36 & $\mathrm{f} 128$ & 821 & f7 & 7511 & 11 & & \\
\hline S & 54 & 1.00 & BEND & 30 & 11 & 29 & $\mathrm{HCH}$ & 106.47 & f1571 & 19 & f156 & 601 & $\mathrm{f} 1$ & 555 & 13 & f1539 & 10 \\
\hline S & 55 & 1.00 & BEND & 31 & 12 & 13 & $\mathrm{HCC}$ & 109.75 & f1368 & 25 & f135 & 562 & f7 & 751 & 10 & & \\
\hline S & 56 & 1.00 & BEND & 32 & 12 & 31 & $\mathrm{HCH}$ & 106.29 & f1571 & 18 & f156 & 671 & $\mathrm{f} 1$ & 549 & 38 & f1547 & 11 \\
\hline s & 57 & 1.00 & BEND & 33 & 13 & 35 & $\mathrm{HCH}$ & 107.90 & f1561 & 29 & f146 & 673 & 31 & & & & \\
\hline S & 58 & 1.00 & BEND & 34 & 13 & 33 & $\mathrm{HCH}$ & 107.91 & f1561 & 44 & f146 & 673 & 31 & & & & \\
\hline S & 59 & 1.00 & BEND & 35 & 13 & 34 & $\mathrm{HCH}$ & 107.84 & f1571 & 16 & f156 & 601 & $\mathrm{f} 1$ & 467 & 28 & & \\
\hline S & 60 & 1.00 & BEND & 36 & 37 & 7 & $\mathrm{OCO}$ & 90.35 & f107 & 70 & f89 1 & 19 & & & & & \\
\hline S & 61 & 1.00 & BEND & 38 & 37 & 36 & $\mathrm{OCO}$ & 177.65 & f 625 & 97 & & & & & & & \\
\hline$S$ & 62 & 1.00 & BEND & 7 & 6 & 5 & OCC & 124.52 & f 453 & 23 & $\mathrm{f} 428$ & 13 & f346 & $5 \quad 14$ & & & \\
\hline s & 63 & 1.00 & BEND & 8 & 6 & 7 & OCO & 123.64 & f 643 & 23 & f346 & 19 & & & & & \\
\hline s & 64 & 1.00 & BEND & 6 & 5 & 4 & $\mathrm{CCC}$ & 109.92 & f243 & 30 & & & & & & & \\
\hline S & 65 & 1.00 & BEND & 10 & 9 & 8 & $\mathrm{CCO}$ & 110.77 & f518 & 30 & f290 & 19 & & & & & \\
\hline S & 66 & 1.00 & BEND & 1 & 2 & 3 & $\mathrm{CCC}$ & 112.50 & f 406 & 42 & f193 & 10 & & & & & \\
\hline S & 67 & 1.00 & BEND & 4 & 3 & 2 & $\mathrm{CCC}$ & 113.14 & $\mathrm{f} 406$ & 14 & $\mathrm{f} 243$ & 10 & f193 & 319 & & & \\
\hline S & 68 & 1.00 & BEND & 11 & 10 & 9 & $\mathrm{CCC}$ & 113.28 & f518 & 13 & f164 & 20 & & & & & \\
\hline $\mathrm{s}$ & 69 & 1.00 & BEND & 12 & 11 & 10 & $\mathrm{CCC}$ & 112.78 & f 408 & 13 & f290 & 18 & f164 & 18 & & & \\
\hline
\end{tabular}




\begin{tabular}{|c|c|c|c|c|c|c|c|c|c|c|c|c|c|c|c|}
\hline $\mathrm{S}$ & 70 & 1.00 & BEND & 13 & 12 & 11 & $\mathrm{CCC}$ & 112.63 & f 408 & f164 & \multicolumn{5}{|l|}{12} \\
\hline S & 71 & 1.00 & BEND & 5 & 4 & 3 & $\mathrm{CCC}$ & 112. & f 428 & 28 & & & & & \\
\hline$S$ & 72 & 1.00 & BEND & 9 & 8 & 6 & $\mathrm{COC}$ & 115 . & f346 & $\mathrm{f} 290$ & $\mathrm{f} 176$ & 13 & & & \\
\hline S & 73 & 1.00 & BEND & 37 & 7 & 6 & $\mathrm{COC}$ & 126. & f80 & 15036 & $5 \quad \mathrm{f} 1919$ & & & & \\
\hline S & 74 & 1.00 & TORS & 14 & 1 & 2 & 3 & $\mathrm{HCCC}$ & -180.04 & $\mathrm{f} 156011$ & f265 2 & & & & \\
\hline S & 75 & 1.00 & TORS & 15 & 1 & 2 & 3 & $\mathrm{HCCC}$ & 59.75 & $\mathrm{f} 128413$ & $\mathrm{f} 1162$ & 10 & f956 & 12 & $\mathrm{f} 26517$ \\
\hline $\mathrm{S}$ & 76 & 1.00 & TORS & 16 & 1 & 2 & 3 & $\mathrm{HCCC}$ & -59.84 & & & & & & \\
\hline S & 77 & 1.00 & TORS & 17 & 2 & 3 & 4 & $\mathrm{HCCC}$ & 56.70 & f763 22 & & & & & \\
\hline S & 78 & 1.00 & TORS & 18 & 2 & 3 & 4 & $\mathrm{HCCC}$ & -59.24 & $\mathrm{f} 144810$ & f1284 & 13 & & & \\
\hline$S$ & 79 & 1.00 & TORS & 19 & 3 & 4 & 5 & $\mathrm{HCCC}$ & 54.39 & $\mathrm{f} 144815$ & f7631 & & & & \\
\hline $\begin{array}{l}\mathrm{S} \\
1\end{array}$ & 80 & 1.00 & TORS & 20 & 3 & 4 & 5 & $\mathrm{HCCC}$ & -61.88 & $\mathrm{f} 144819$ & f1372 & 15 & f1334 & 412 & $\mathrm{f} 1284$ \\
\hline $\mathrm{S}$ & 81 & 1.00 & TORS & 21 & 4 & 5 & 6 & $\mathrm{HCCC}$ & -178.66 & $\mathrm{f} 140510$ & f721 1 & & & & \\
\hline $\mathrm{S}$ & 82 & 1.00 & TORS & 22 & 4 & 5 & 6 & $\mathrm{HCCC}$ & -62.72 & $\mathrm{f} 140521$ & f1350 & 13 & & & \\
\hline S & 83 & 1.00 & TORS & 23 & 5 & 6 & 7 & $\mathrm{HCCO}$ & -56.19 & $\mathrm{f} 140811$ & & & & & \\
\hline $\begin{array}{l}S \\
1\end{array}$ & 84 & 1.00 & TORS & 24 & 5 & 6 & 7 & $\mathrm{HCCO}$ & -174.43 & $\mathrm{f} 141510$ & f1408 & 10 & $\mathrm{f} 1405$ & 513 & $\mathrm{f} 1230$ \\
\hline S & 85 & 1.00 & TORS & 25 & 9 & 8 & 6 & $\mathrm{HCOC}$ & -202.65 & $\mathrm{f} 144525$ & & & & & \\
\hline $\mathrm{S}$ & 86 & 1.00 & TORS & 26 & 9 & 8 & 6 & $\mathrm{HCOC}$ & 40.42 & $\mathrm{f} 144522$ & & & & & \\
\hline $\mathrm{S}$ & 87 & 1.00 & TORS & 27 & 10 & 11 & 12 & $\mathrm{HCCC}$ & 54.60 & $\mathrm{f} 1451 \quad 10$ & & & & & \\
\hline S & 88 & 1.00 & TORS & 28 & 10 & 11 & 12 & $\mathrm{HCCC}$ & -62.70 & f1451 12 & $\mathrm{f} 1217$ & 14 & & & \\
\hline $\mathrm{S}$ & 89 & 1.00 & TORS & 29 & 11 & 12 & 13 & $\mathrm{HCCC}$ & 58.87 & f1451 13 & $\mathrm{f} 7511$ & & & & \\
\hline S & 90 & 1.00 & TORS & 30 & 11 & 12 & 13 & $\mathrm{HCCC}$ & -57.34 & $\mathrm{f} 145118$ & f1356 & 14 & & & \\
\hline S & 91 & 1.00 & TORS & 31 & 12 & 11 & 10 & $\mathrm{HCCC}$ & 58.51 & f751 19 & & & & & \\
\hline $\mathrm{S}$ & 92 & 1.00 & TORS & 32 & 12 & 11 & 10 & $\mathrm{HCCC}$ & -57.34 & $\mathrm{f} 141512$ & f1347 & 10 & & & \\
\hline S & 93 & 1.00 & TORS & 33 & 13 & 12 & 11 & $\mathrm{HCCC}$ & -179.92 & $\mathrm{f} 156112$ & f2593 & & & & \\
\hline S & 94 & 1.00 & TORS & 34 & 13 & 12 & 11 & $\mathrm{HCCC}$ & 59.96 & f259 22 & & & & & \\
\hline S & 95 & 1.00 & TORS & 35 & 13 & 12 & 11 & $\mathrm{HCCC}$ & -59.70 & f1171 14 & f960 1 & $\mathrm{f} 2$ & 259 & 17 & \\
\hline $\mathrm{S}$ & 96 & 1.00 & TORS & 36 & 37 & 7 & 6 & OCOC & -186.82 & $\mathrm{f} 1284$ & & & & & \\
\hline S & 97 & 1.00 & TORS & 7 & 6 & 5 & 4 & $\mathrm{OCCC}$ & 63.35 & f38 29 & $\mathrm{f} 29 \quad 13$ & & & & \\
\hline$S$ & 98 & 1.00 & TORS & 6 & 5 & 4 & 3 & $\mathrm{CCCC}$ & 59.36 & $\mathrm{f} 13811$ & f69 12 & f50 & 21 & f29 & 10 \\
\hline $\mathrm{S}$ & 99 & 1.00 & TORS & 10 & 9 & 8 & 6 & $\mathrm{CCOC}$ & -82.65 & f123 12 & f4328 & & & & \\
\hline S & 100 & 1.00 & TORS & 1 & 2 & 3 & 4 & $\mathrm{CCCC}$ & -181.35 & $\mathrm{f} 13827$ & $\mathrm{f123} 14$ & & & & \\
\hline S & 101 & 1.00 & TORS & 11 & 10 & 9 & 8 & $\mathrm{CCCO}$ & -59.22 & $\mathrm{f} 17622$ & f69 12 & f4 3 & 18 & & \\
\hline S & 102 & 1.00 & TORS & 12 & 11 & 10 & 9 & $\mathrm{CCCC}$ & -183.51 & f123 13 & f84 10 & f69 & 14 & f43 & 19 \\
\hline$S$ & 103 & 1.00 & TORS & 13 & 12 & 11 & 10 & $\mathrm{CCCC}$ & -179.15 & f176 10 & $\mathrm{f} 12314$ & f84 & 417 & f69 & 10 \\
\hline S & 104 & 1.00 & TORS & 5 & 4 & 3 & 2 & $\mathrm{CCCC}$ & -183.31 & f89 16 & f84 24 & f69 1 & 12 & & \\
\hline $\mathrm{S}$ & 105 & 1.00 & TORS & 9 & 8 & 6 & 5 & $\mathrm{COCC}$ & -183.45 & $\mathrm{f} 12311$ & f38 20 & f29 & 11 & f19 & 11 \\
\hline S & 106 & 1.00 & TORS & 37 & 7 & 6 & 5 & $\mathrm{COCC}$ & 23.15 & f29 34 & f19 39 & & & & \\
\hline S & 107 & 1.00 & OUT & 38 & 7 & 36 & 37 & OOOC & 0.03 & f64499 & & & & & \\
\hline$S$ & 108 & 1.00 & OUT & 8 & 5 & 7 & 6 & OCOC & 1.82 & f721 23 & f643 12 & & & & \\
\hline
\end{tabular}

37 STRETCHING modes:

$\begin{array}{llllllllllllllllllll}1 & 2 & 3 & 4 & 5 & 6 & 7 & 8 & 9 & 10 & 11 & 12 & 13 & 14 & 15 & 16 & 17 & 18 & 19 & 20\end{array}$

$\begin{array}{lllllllllllllllll}21 & 22 & 23 & 24 & 25 & 26 & 27 & 28 & 29 & 30 & 31 & 32 & 33 & 34 & 35 & 36 & 37\end{array}$

36 BENDING modes:

$\begin{array}{llllllllllllllllllll}38 & 39 & 40 & 41 & 42 & 43 & 44 & 45 & 46 & 47 & 48 & 49 & 50 & 51 & 52 & 53 & 54 & 55 & 56 & 57\end{array}$

$\begin{array}{llllllllllllllll}58 & 59 & 60 & 61 & 62 & 63 & 64 & 65 & 66 & 67 & 68 & 69 & 70 & 71 & 72 & 73\end{array}$

35 TORSION modes:

$\begin{array}{llllllllllllllllllll}74 & 75 & 76 & 77 & 78 & 79 & 80 & 81 & 82 & 83 & 84 & 85 & 86 & 87 & 88 & 89 & 90 & 91 & 92 & 93\end{array}$

$949596979899100101 \quad 102 \quad 103 \quad 104 \quad 105 \quad 106 \quad 107 \quad 108$

3 OUT-OF-PLANE BENDING (WAGGING) modes

107108

$66 \mathrm{CH}$ modes:

$\begin{array}{llllllllllllllllllll}1 & 2 & 3 & 4 & 5 & 6 & 7 & 8 & 9 & 10 & 11 & 12 & 13 & 14 & 15 & 16 & 17 & 18 & 19 & 20\end{array}$

$\begin{array}{llllllllllllllllllll}21 & 22 & 38 & 39 & 40 & 41 & 42 & 43 & 44 & 45 & 46 & 47 & 48 & 49 & 50 & 51 & 52 & 53 & 54 & 55\end{array}$

$\begin{array}{llllllllllllllllllll}56 & 57 & 58 & 59 & 74 & 75 & 76 & 77 & 78 & 79 & 80 & 81 & 82 & 83 & 84 & 85 & 86 & 87 & 88 & 89\end{array}$

$90 \quad 9192 \quad 93 \quad 94 \quad 95$ 


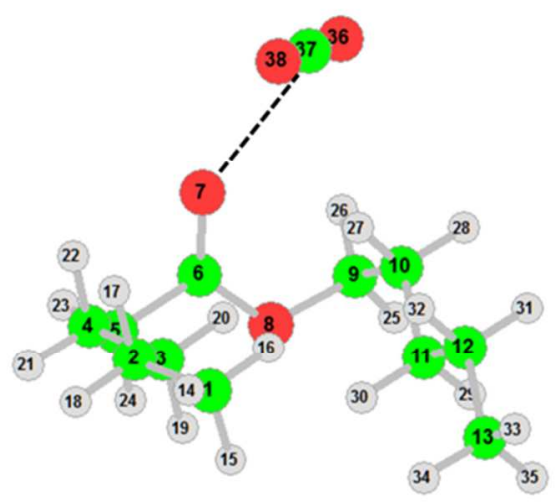

Figure S4. Atom numbering for the pentylhexanoate/ $\mathrm{CO}_{2}$ complex from the ester-side starting structure. Atom colour code: Green=C; grey=H; red=O.

Table s3. Definition of internal coordinates (natural) and their main contributions to the $P E D$ (values $\leq 10$ \% neglected) for the pentylhexanoate/ $\mathrm{H}_{2} \mathrm{O}$ complex from the ester-side starting structure. The indicated frequencies are unscaled.

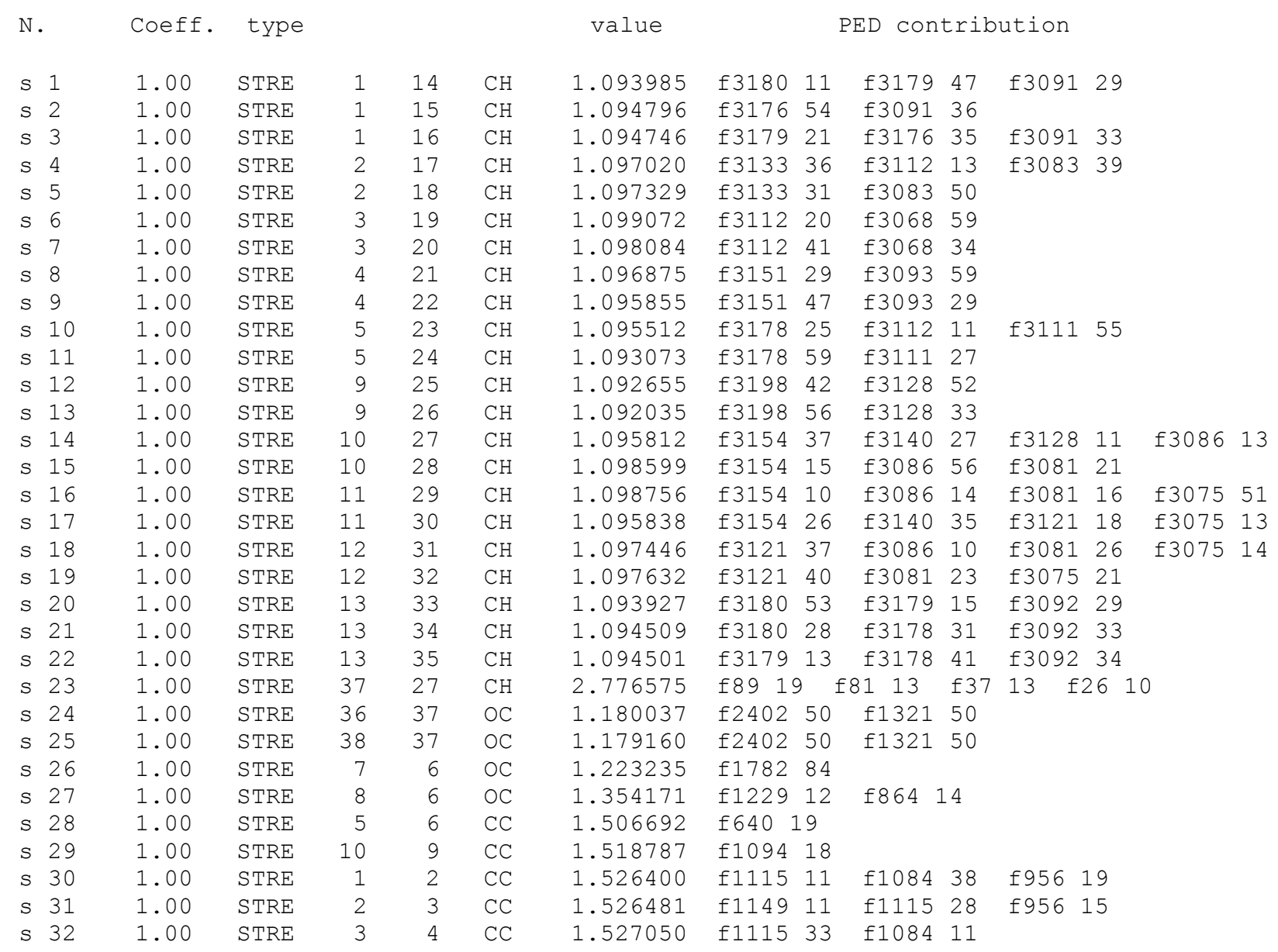




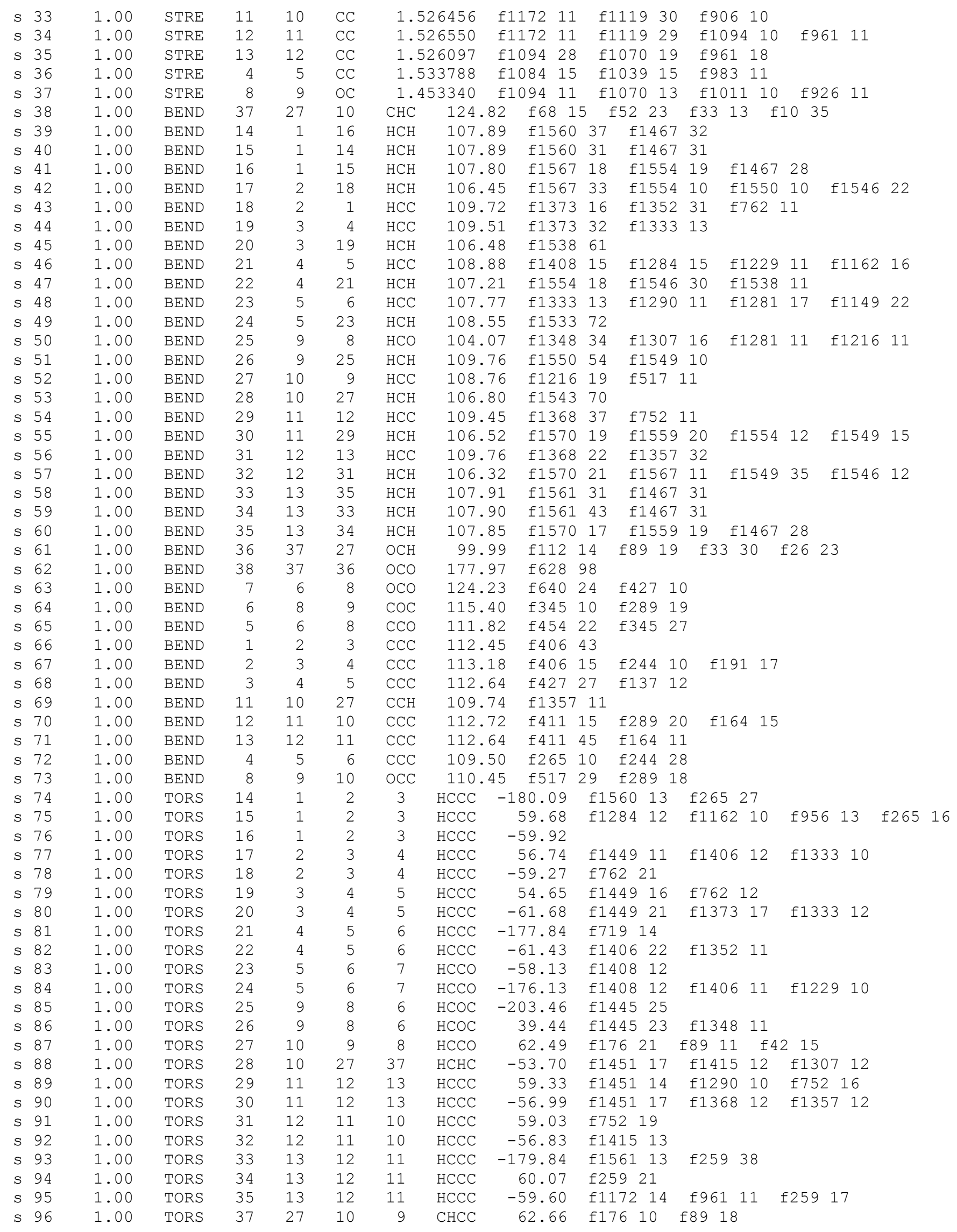




\begin{tabular}{|c|c|c|c|c|c|c|c|c|c|c|c|c|c|}
\hline S & 97 & 1.00 & TORS & 36 & 37 & 27 & 10 & $\mathrm{OCHC}$ & -4.75 & $\mathrm{f} 11232$ & $\mathrm{f75} 10$ & 12622 & \\
\hline S & 98 & 1.00 & TORS & 7 & 6 & 8 & 9 & $\mathrm{OCOC}$ & -2.43 & f37 17 & $\mathrm{f10} 13$ & & \\
\hline$S$ & 99 & 1.00 & TORS & 6 & 8 & 9 & 10 & $\mathrm{COCC}$ & -83.73 & $\mathrm{f} 12015$ & $\mathrm{f} 112 \quad 14$ & f42 14 & $\mathrm{f} 10$ \\
\hline$S$ & 100 & 1.00 & TORS & 1 & 2 & 3 & 4 & $\mathrm{CCCC}$ & -181.38 & $\mathrm{f} 13725$ & $\mathrm{f52} 10$ & & \\
\hline S & 101 & 1.00 & TORS & 2 & 3 & 4 & 5 & $\mathrm{CCCC}$ & -183.01 & f81 11 & f75 40 & & \\
\hline S & 102 & 1.00 & TORS & 3 & 4 & 5 & 6 & $\mathrm{CCCC}$ & 60.15 & $\mathrm{f} 191 \quad 11$ & $\mathrm{f} 13711$ & f68 11 & $\mathrm{f} 52$ \\
\hline$S$ & 103 & 1.00 & TORS & 11 & 10 & 27 & 37 & $\mathrm{CCHC}$ & -172.88 & $\mathrm{f} 17615$ & f37 17 & f26 11 & \\
\hline S & 104 & 1.00 & TORS & 12 & 11 & 10 & 9 & $\mathrm{CCCC}$ & -183.48 & $\mathrm{f} 12021$ & f81 19 & f68 19 & $\mathrm{f} 42$ \\
\hline S & 105 & 1.00 & TORS & 13 & 12 & 11 & 10 & $\mathrm{CCCC}$ & -178.61 & $\mathrm{f} 17611$ & f120 15 & f81 19 & \\
\hline S & 106 & 1.00 & TORS & 4 & 5 & 6 & 7 & $\mathrm{CCCO}$ & 61.61 & f33 25 & f26 19 & f10 10 & \\
\hline S & 107 & 1.00 & OUT & 38 & 7 & 36 & 37 & O००C & 0.47 & f64588 & & & \\
\hline S & 108 & 1.00 & OUT & 5 & 7 & 8 & 6 & $\mathrm{COOC}$ & 1.95 & f719 22 & & & \\
\hline
\end{tabular}

37 STRETCHING modes:

$\begin{array}{llllllllllllllllllll}1 & 2 & 3 & 4 & 5 & 6 & 7 & 8 & 9 & 10 & 11 & 12 & 13 & 14 & 15 & 16 & 17 & 18 & 19 & 20\end{array}$

$\begin{array}{lllllllllllllllll}21 & 22 & 23 & 24 & 25 & 26 & 27 & 28 & 29 & 30 & 31 & 32 & 33 & 34 & 35 & 36 & 37\end{array}$

36 BENDING modes:

$\begin{array}{llllllllllllllllllll}38 & 39 & 40 & 41 & 42 & 43 & 44 & 45 & 46 & 47 & 48 & 49 & 50 & 51 & 52 & 53 & 54 & 55 & 56 & 57\end{array}$

$\begin{array}{llllllllllllllll}58 & 59 & 60 & 61 & 62 & 63 & 64 & 65 & 66 & 67 & 68 & 69 & 70 & 71 & 72 & 73\end{array}$

35 TORSION modes:

$\begin{array}{llllllllllllllllllll}74 & 75 & 76 & 77 & 78 & 79 & 80 & 81 & 82 & 83 & 84 & 85 & 86 & 87 & 88 & 89 & 90 & 91 & 92 & 93\end{array}$

$94 \quad 95969798 \quad 99 \quad 100 \quad 101 \quad 102 \quad 103 \quad 104 \quad 105 \quad 106 \quad 107 \quad 108$

2 OUT-OF-PLANE BENDING (WAGGING) modes

107108

$71 \mathrm{CH}$ modes:

$\begin{array}{llllllllllllllllllll}1 & 2 & 3 & 4 & 5 & 6 & 7 & 8 & 9 & 10 & 11 & 12 & 13 & 14 & 15 & 16 & 17 & 18 & 19 & 20\end{array}$

$\begin{array}{llllllllllllllllllll}21 & 22 & 38 & 39 & 40 & 41 & 42 & 43 & 44 & 45 & 46 & 47 & 48 & 49 & 50 & 51 & 52 & 53 & 54 & 55\end{array}$

$\begin{array}{llllllllllllllllllll}56 & 57 & 58 & 59 & 60 & 69 & 74 & 75 & 76 & 77 & 78 & 79 & 80 & 81 & 82 & 83 & 84 & 85 & 86 & 87\end{array}$

$\begin{array}{lllllllllll}88 & 89 & 90 & 91 & 92 & 93 & 94 & 95 & 96 & 97 & 103\end{array}$

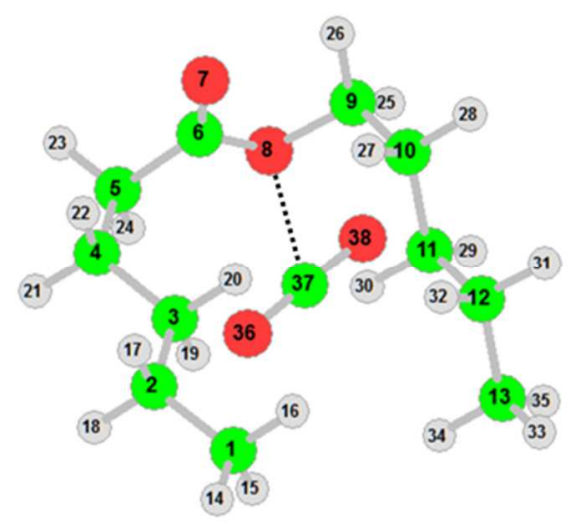

Figure S5. Atom numbering for the pentylhexanoate/ $\mathrm{CO}_{2}$ complex from the $\mathrm{Sp}^{3}-\mathrm{O} / \mathrm{CO}_{2}$ starting structure. Atom colour code: Green=C; grey=H; red $=0$.

Table s4. Definition of internal coordinates (natural) and their main contributions to the $P E D$ (values $\leq 10$ \% neglected) for the pentylhexanoate/ $\mathrm{H}_{2} \mathrm{O}$ complex from the ether-side starting structure. The indicated frequencies are unscaled. 


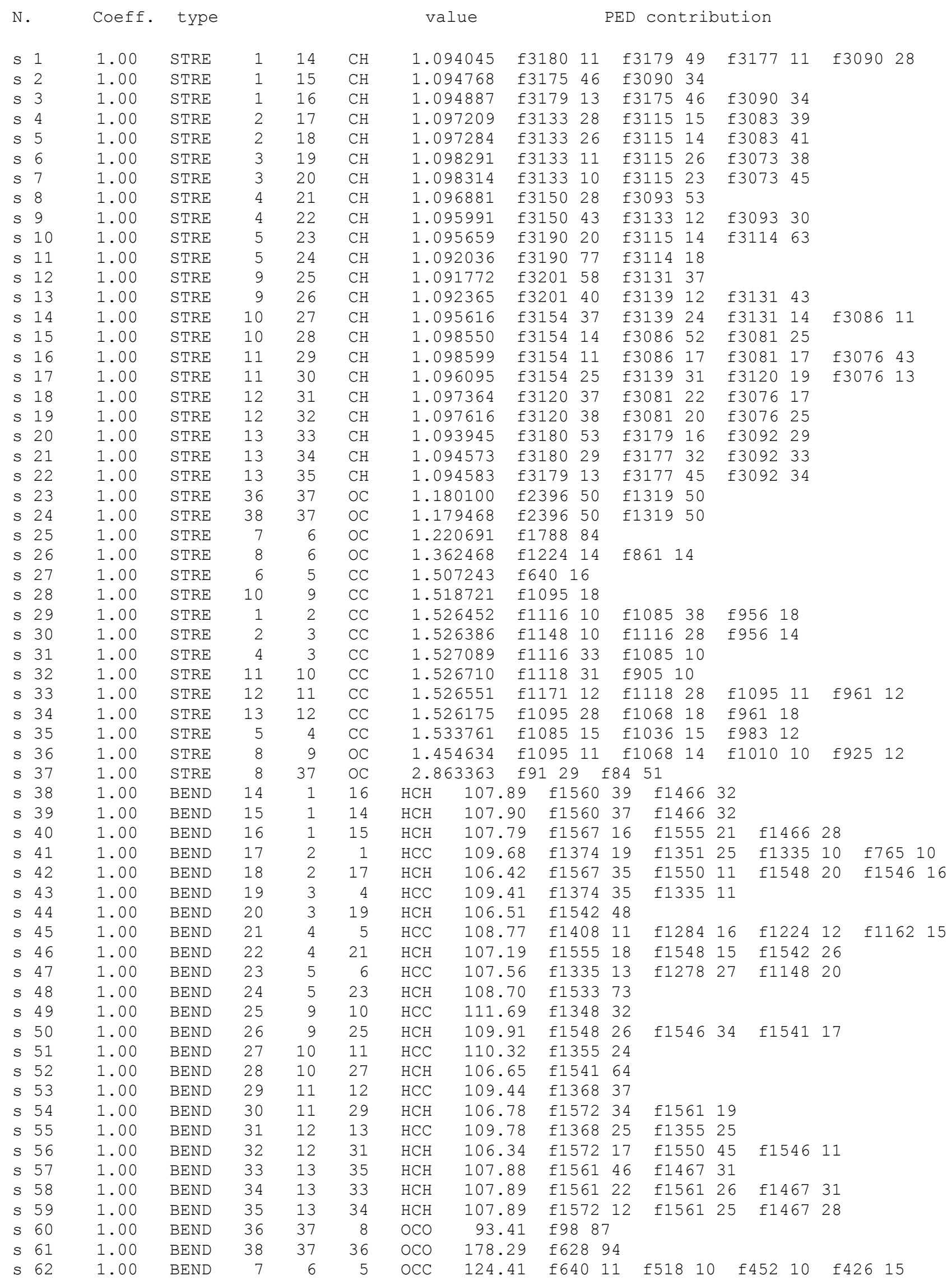




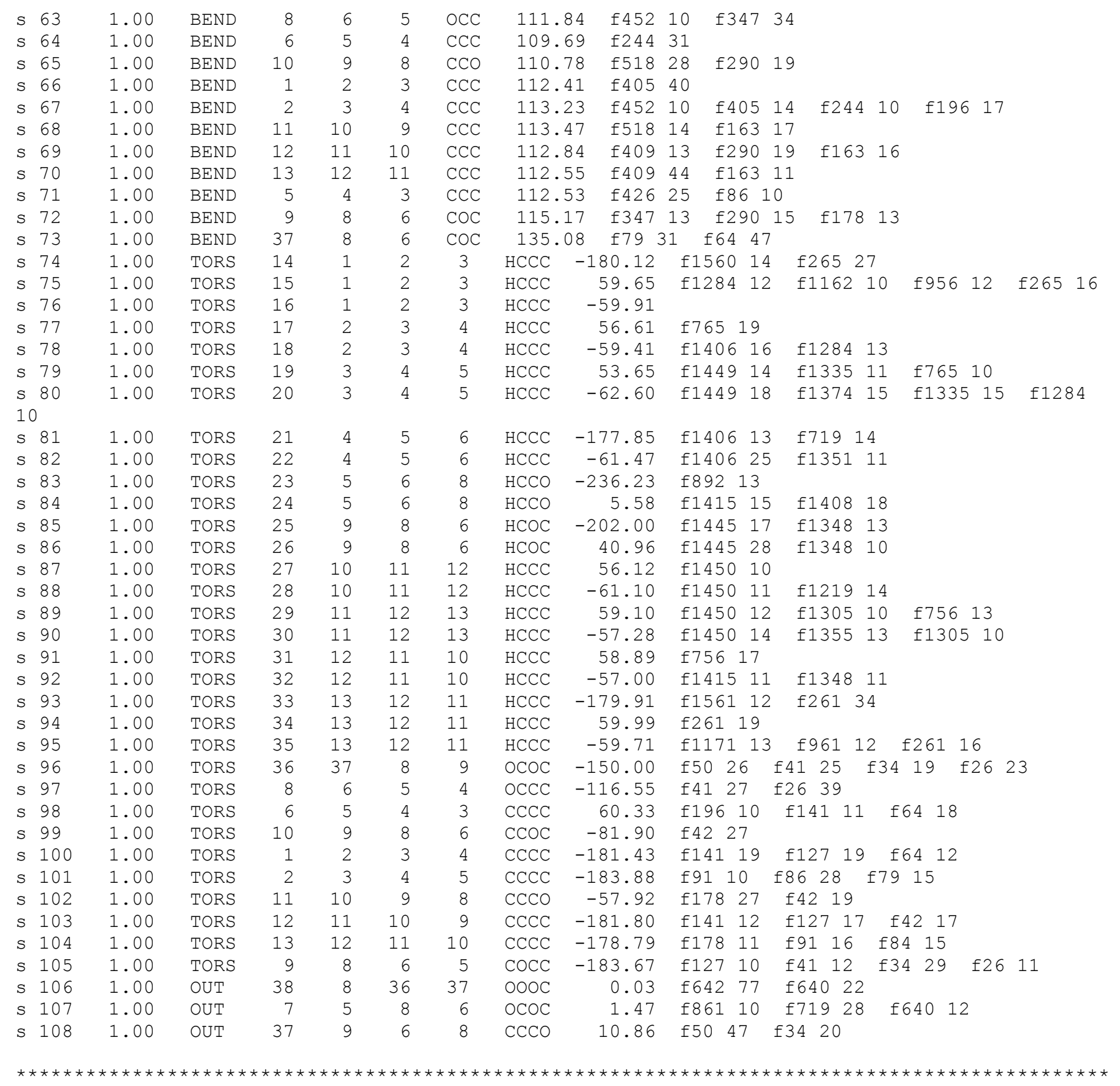

37 STRETCHING modes:

$\begin{array}{llllllllllllllllllll}1 & 2 & 3 & 4 & 5 & 6 & 7 & 8 & 9 & 10 & 11 & 12 & 13 & 14 & 15 & 16 & 17 & 18 & 19 & 20\end{array}$

$\begin{array}{lllllllllllllllll}21 & 22 & 23 & 24 & 25 & 26 & 27 & 28 & 29 & 30 & 31 & 32 & 33 & 34 & 35 & 36 & 37\end{array}$

36 BENDING modes:

$\begin{array}{llllllllllllllllllll}38 & 39 & 40 & 41 & 42 & 43 & 44 & 45 & 46 & 47 & 48 & 49 & 50 & 51 & 52 & 53 & 54 & 55 & 56 & 57\end{array}$

$\begin{array}{llllllllllllllll}58 & 59 & 60 & 61 & 62 & 63 & 64 & 65 & 66 & 67 & 68 & 69 & 70 & 71 & 72 & 73\end{array}$

35 TORSION modes:

$\begin{array}{llllllllllllllllllll}74 & 75 & 76 & 77 & 78 & 79 & 80 & 81 & 82 & 83 & 84 & 85 & 86 & 87 & 88 & 89 & 90 & 91 & 92 & 93\end{array}$

$949596979899100101 \quad 102 \quad 103 \quad 104 \quad 105 \quad 106 \quad 107108$

3 OUT-OF-PLANE BENDING (WAGGING) MODES

106107108 
$66 \mathrm{CH}$ modes:

$\begin{array}{lllllllllllllllllllll}1 & 2 & 3 & 4 & 5 & 6 & 7 & 8 & 9 & 10 & 11 & 12 & 13 & 14 & 15 & 16 & 17 & 18 & 19 & 20\end{array}$

$\begin{array}{llllllllllllllllllll}21 & 22 & 38 & 39 & 40 & 41 & 42 & 43 & 44 & 45 & 46 & 47 & 48 & 49 & 50 & 51 & 52 & 53 & 54 & 55\end{array}$

$\begin{array}{llllllllllllllllllll}56 & 57 & 58 & 59 & 74 & 75 & 76 & 77 & 78 & 79 & 80 & 81 & 82 & 83 & 84 & 85 & 86 & 87 & 88 & 89\end{array}$

$\begin{array}{llllll}90 & 91 & 92 & 93 & 94 & 95\end{array}$ 